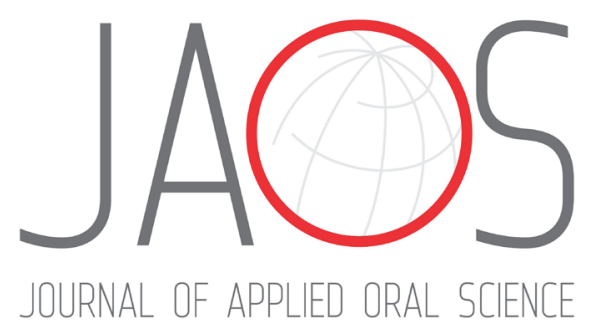

\title{
Effects of ClpP protease on biofilm formation of Enterococcus faecalis
}

\section{Abstract}

Ying FENG ${ }^{1}$

Hongyuan WANG ${ }^{1}$

Lu HE 2

Yi LIU 3

Hong LI ${ }^{1}$ iD
Submitted: August 30, 2020 Modification: October 28, 2020 Accepted: November 25, 2020

Corresponding address: Hong Li

Department of Endodontics - Beijing Stomatological Hospital - School of Stomatology - Capital Medical University - Tian Tan Xi Li No.4 Beijing - 100050 - China Phone: 86-10-57099233 e-mail: endolihong@qq.com
Enterococcus faecalis ( $E$. faecalis), one of the main pathogens responsible for refractory periapical periodontitis and nosocomial infections, exhibits markedly higher pathogenicity in biofilms. Objectives: Studies have shown that caseinolytic protease $\mathrm{P}(\mathrm{ClpP})$ is involved in biofilm formation. However, to date, few studies have investigated the role of ClpP in the survival of $E$. faecalis, and in enhancing biofilm formation. Therefore, we investigated the role of ClpP in the formation of $E$. faecalis biofilms. Methodology: In our study, we used homologous recombination to construct clpP deleted and clpP complement strains of $E$. faecalis ATCC 29212. A viable colony counting method was used to analyze the growth patterns of $E$. faecalis. Crystal violet staining (CV) and confocal scanning laser microscopy (CLSM) were used to characterize biofilm mass formation and scanning electron microscopy (SEM) was used to observe the biofilm microstructure. Data was statistically analyzed via Student's t-test or one-way analysis of variance (ANOVA). Results: The results exhibited altered growth patterns for the clpP deletion strains and depleted polysaccharide matrix, resulting in reduced biofilm formation capacity compared to the standard strains. Moreover, ClpP was observed to increase biofilm formation in E. faecalis. Conclusion: Our study shows that ClpP can increase biofilm formation in $E$. faecalis and emphasizes the importance of ClpP as a potential target against $E$. faecalis.

Key words: Enterococcus faecalis. ClpP protease. Biofilm. Growth.

${ }^{1}$ Department of Endodontics, School of Stomatology, Capital Medical University.

${ }^{2}$ Affiliated Stomatology Hospital of Guangzhou Medical University.

${ }^{3}$ Laboratory of Tissue Regeneration and Immunology and Department of Periodontics, Beijing Key Laboratory of Tooth Regeneration and Function Reconstruction, School of Stomatology, Capital Medical University. 


\section{Introduction}

Enterococcus faecalis (E. faecalis) is one of the primary etiologic pathogens of refractory periapical periodontitis, secondary root canal infections, and pulp infection. It is also the main pathogen of nosocomial infections, such as bacterial endocarditis and meningitis. ${ }^{1-2}$ The ability of $E$. faecalis to form biofilms directly determines its survival, adaptability to the environment and pathogenicity. It was reported that more than $40 \%$ of $E$. faecalis clinical isolates can form biofilms. ${ }^{3}$ E. faecalis is $100-1000$ times more pathogenic in biofilms than in the plankton state, ${ }^{4}$ since biofilms provide protection against environmental stress, including lacks of nutrients, high alkalinity and antibiotics. ${ }^{5}$ In the field of Endodontics, the medicaments used to remove bacteria from the root canal system primarily include calcium hydroxide and antibiotics. However, these agents are associated with poor efficiency and have been shown to cause adverse effects. ${ }^{6}$ Hence, there is currently a lack of effective medication capable of treating $E$. faecalis. Therefore, investigating the mechanism associated with $E$. faecalis biofilm formation is essential to inform the development of effective treatment methods.

Accordingly, the stress response of $E$. faecalis has become the subject of intensive research. The adaptability of $E$. faecalis to stress and crossimmunoprotectivity is associated with increased protein synthesis. However, except for a few stressrelated proteins, such as Gls24, and the molecular chaperones, GroEL and DnaK, most proteins involved in the stress response remain unknown. ${ }^{7-8}$ Caseinolytic protease $\mathrm{P}$ (ClpP) is an ATP-dependent proteolytic enzyme that plays important roles in bacteria, parasites, and human mitochondria. Specifically, ClpP participates in the hydrolysis of misfolded and defective proteins, whereas $C l p P$ is an important gene involved in biofilm formation. ${ }^{9-18}$

Recent studies have reported that CIpP exerts different effects on bacterial biofilm formation. However, the general consensus is that ClpP enhances biofilm formation, since the biofilm formation of Streptococcus mutans, Actinobacillus pleuropneumoniae, and Bacillus subtilis decrease when ClpP was mutated. ${ }^{12-14}$ Our previous study found that ClpP of $P$. gingivalis improved biofilm formation by regulating adhesion-related factors and the density-sensing system. ${ }^{15}$ Meanwhile, other studies have shown that ClpP inhibits biofilm formation. For example, studies reported that ClpP inhibits biofilm formation by regulating Sle1 and agr in Staphylococcus aureus. ${ }^{16}$ In fact, researchers hold different views on the role of $c l p P$ in the formation of biofilm for same bacteria. Shanks, et al. ${ }^{17}$ (2006) found that when $c l p P$ was mutated, the biofilm of Pseudomonas aeruginosa increased; however, Hall, et al. ${ }^{18}$ (2017) stated that $c l p P$ enhanced its ability to form biofilms.

To date, few studies have investigated the role of ClpP in the survival of $E$. faecalis or its ability to influence biofilm formation. E. faecalis has a unique ability to tolerate hypoxia, starvation, and high alkalinity in reinfected root canals making it challenging to treat with current antibiotics. Hence, identifying effective antibiotic targets is an urgent concern for this pathogen. In our study, we investigated the role of ClpP in the formation of $E$. faecalis biofilms. These results advance the current understanding on the role of ClpP in E. faecalis biofilm formation and may lead to novel antibacterial therapies in the future.

\section{Methodology}

\section{Bacterial culture conditions and plasmids}

The E. faecalis standard strains ATCC 29212 (Manassas, VA, USA) was cultured in brain heart infusion medium (BHI, Oxoid Deutschland $\mathrm{GmbH}$, Wesel, Germany) in an anaerobic environment at $37^{\circ} \mathrm{C}$. BHI plates contained $\mathrm{v} / \mathrm{v}$ vitamin $\mathrm{K}$, hemin, and $5 \mathrm{~g}$ yeast extract/L (Sigma Aldrich). Escherichia coli strains JM109 was grown in Luria-Bertani medium. Antibiotics were purchased from Sigma Chemical Co.

\section{Construction of deletion and complemented strains}

Based on the strains ATCC 29212 chromosomal DNA sequence, primers for the $c / p P$ gene were designed (Figure 1). PCR was used to amplify the upstream and downstream homologous fragments, and the product was cloned into plasmid pUC18. Based on the ermB gene sequence of plasmid pJRS233, the erm $B$ gene fragment was amplified and inserted into the recombinant plasmid pUC18- $\triangle c / p P$. pUC18- $\triangle c l p P$, pUC18- $\triangle c l p P$-ermB, and pUC18- $\triangle c l p P$-ermB-clpP were transformed into $E$. coli strains JM109 and selected for with ampicillin. The $c l p P$ deletion and complement plasmids were then transformed into $E$. faecalis. PCR and enzyme-cutting electrophoresis were used 


\begin{tabular}{|c|c|c|}
\hline Name & Sequence (5'-3') & dsDNA \\
\hline$\Delta$ clpP-F1 & CCCAAGCTTTCTTCAATTAAGTACGTCGG & 29 \\
\hline$\Delta$ clpP-R1 & AGgCCCGGGTTAGCTGCAGAAAAAAGTAAATCCTCCTATATAA & 43 \\
\hline$\Delta$ clpP-F2 & TTTTCTGCAGCTAACCCGGGGGAAAATAAAAAAATGGAAACAGCG & 45 \\
\hline$\Delta$ clpP-R2 & CCGGAATTGGGCTTCACATTGGTGAGAG & 48 \\
\hline ermB-F & AAAACTGCAGCGAGCTCGAATTACG & 25 \\
\hline
\end{tabular}

Figure 1- Primers used for PCR

to identify the accuracy of the sequence, and highexpression strains were selected using media.

\section{Growth analysis of E. faecalis}

Frozen E. faecalis ATCC29212 was added to $5 \mathrm{~mL}$ of $\mathrm{BHI}$ broth and cultured under anaerobic conditions at $37^{\circ} \mathrm{C}$ for $16 \mathrm{~h}$. Next, $500 \mu \mathrm{L}$ of bacterial solution $(1.5 \times$ $10^{9} \mathrm{CFU} / \mathrm{mL}$ ) was added to $50 \mathrm{~mL}$ of $\mathrm{BHI}$ medium, from which $1 \mathrm{~mL}$ of bacterial solution was serially diluted and cultured under anaerobic conditions at $37^{\circ} \mathrm{C}$. From $0 \mathrm{~h}$ the culture was observed every $2.5 \mathrm{~h}$ for a total of $25 \mathrm{~h}$. The cultures were also observed every $12 \mathrm{~h}$ for a total of $61 \mathrm{~h}$. Bacterial growth was recorded using the viable colony count method.

\section{Construction of the biofilm model}

E. faecalis standard, mutant, and complementary strains were inoculated in $\mathrm{BHI}$ broth. After anaerobic culture at $37^{\circ} \mathrm{C}$ for $24 \mathrm{~h}$, independent colonies were selected in logarithmic phase and cultured in $\mathrm{BHI}$ liquid medium at $37^{\circ} \mathrm{C}$. The bacterial density was then adjusted to $10^{5} \mathrm{CFU} / \mathrm{mL}$. In a sterile environment, suitable size coverslips were placed in 12-well plates and $1 \mathrm{~mL}$ of $E$. faecalis suspension was added. One tablet was used for each strain. Plates were incubated at $37^{\circ} \mathrm{C}$ for $6,12,24$, and $48 \mathrm{~h}$. The experiments were repeated independently in triplicate for each time point and with each strain.

\section{Crystal violet staining}

Crystal violet (CV) staining was performed to quantify the biofilm mass. The biofilms were constructed using 12-well polystyrene tablets. One tablet was used for each strain. The culture samples were fixed with $200 \mu \mathrm{L} 1 \%$ paraformaldehyde for 15 min and washed three times with $200 \mu \mathrm{L}$ phosphate buffered saline (PBS). Subsequently, $200 \mu \mathrm{L}$ of $0.01 \%$ $\mathrm{CV}$ was added for $15 \mathrm{~min}$, and $200 \mu \mathrm{L}$ PBS was used for clearing. After mixing with $200 \mu \mathrm{L}$ of ethanol and acetone for $5 \mathrm{~min}$, the product was transferred to a 96-well plate and OD 570 readings were obtained for quantification. Three wells were used for each sample at each observation time point. The observation times were at $6,12,24$, and $48 \mathrm{~h}$. Each assay was repeated three times.

\section{Confocal scanning laser microscopy}

Confocal scanning laser microscopy (CLSM) was performed to determine $E$. faecalis cell viability and to observe the attached bacterial areas. Each strain was cultured under anaerobic conditions at $37^{\circ} \mathrm{C}$ for $6,12,24$, and $48 \mathrm{~h}$. A total of $200 \mu \mathrm{L}$ SYT09 and PI fluorescent dyes were added to samples in the dark for $15 \mathrm{~min}$ at $37^{\circ} \mathrm{C}$. In addition, FITC-ConA and PI were used to stain the polysaccharide matrix. The processed biofilms were observed using the CLSM system (TCS SP5; Leica Microsystems, Germany). Live and dead bacteria emitted green and red fluorescein, respectively. An image-processing program (Leica Microsystems) was used for digital reconstruction.

\section{Scanning electron microscopy}

Scanning electron microscopy (SEM) was performed to observe the biofilm microstructure. Three samples were rinsed with PBS after $24 \mathrm{~h}$, fixed in $2.5 \%$ glutaraldehyde for $2 \mathrm{~h}$, and dehydrated in a graded series of ethanol (30\%-100\%). Isoamyl acetate was used to reach the critical point of drying, and ion sputter (108 Auto; Cressington, Watford, UK) was used for observation.

\section{Statistical analysis}

All experiments were repeated at least three times. All analyses were performed using SPSS 18.0 statistical software (Superior Performing Software Systems, Chicago, USA). Student's t-test or oneway analysis of variance (ANOVA) was performed to conduct the statistical analysis. $\mathrm{P}<0.05$ was considered statistically significant. 


\section{Results}

\section{Construction of $E$. faecalis $\Delta \mathrm{clpP}$ mutant strains and complement strains}

Upstream and downstream primers for the $c / p P$ gene were used in a PCR-based overlap extension method to obtain the $\triangle c l p P$ fragment (1096 bp). The results are shown in Figure $2 \mathrm{a}$. Next, the $\Delta c l p P$ fragment was cloned into the plasmid pUC18 to generate $p U C 18-\triangle c l p P$. The recombinant plasmid pUC18- $\triangle$ clpP was verified by PCR and enzymatic digestion, and the target bands of 1096 bp and 2686 bp were obtained (Figure $2 \mathrm{~d}$ ). The erythromycin resistance gene ermB was inserted into $p U C 18-\triangle c l p P$ to create $p U C 18-\triangle c l p P$-ermB, which was verified by PstI and SmaI digestion to produce the 1705 bp and 3782 bp target bands (Figure 2c). Additionally, clpP was inserted into $p U C 18-\triangle c / p P$-ermB to generate pUC18- $\triangle c l p P$-ermB-clpP (Figure 2b). Recombinant plasmids $p U C 18-\triangle c l p P$-ermB and $p U C 18-\triangle c l p P$-ermB$c / p P$ were transformed into $E$. faecalis competent cells. After screening for positive clones, the final $\triangle c l p P$ mutant strains and complemented strains were obtained (Figure 2e and $2 \mathrm{f}$ ).

\section{ClpP protease deletion inhibited growth of $E$. faecalis}

As shown in Figure 3a, all three strains entered the logarithmic growth phase after $4 \mathrm{~h}$ and reached a peak at $14 \mathrm{~h}$. The growth curves of the standard and $c / p P$ complement strains were similar. The quiescent stage was approximately $10 \mathrm{~h}-24 \mathrm{~h}$, and gradually decreased thereafter. The quiescent stage of the $\Delta c l p P$ mutant strains was $12 \mathrm{~h}-14 \mathrm{~h}$, and the duration was shorter. The $\triangle c l p P$ mutant strains exhibited impaired growth compared with the standard and clpP complement strains $(p<0.05)$. This result indicates an important

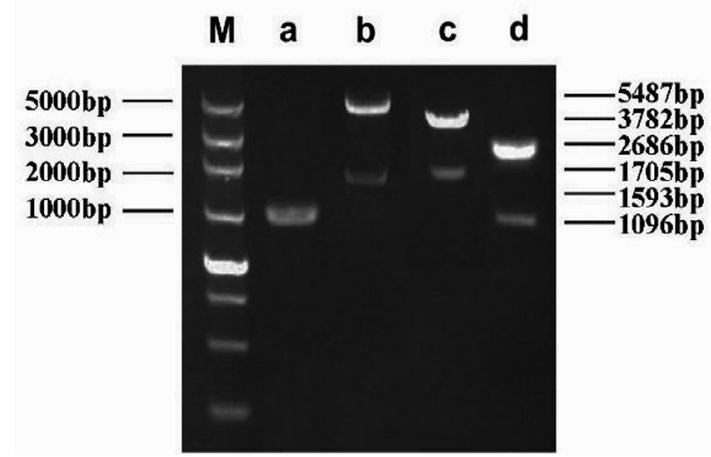

role for ClpP protease in the optimal growth of $E$. faecalis (Figure 3a).

\section{ClpP protease deletion inhibited biofilm mass}

The three strains were incubated for $6,12,24$, and $48 \mathrm{~h}$ for biofilm formation analysis. CV staining showed that the biofilm initially formed after culturing the strains for $6 \mathrm{~h}$. With increased culture time, the biofilm mass also gradually increased. A mature biofilm was observed at $48 \mathrm{~h}$. Compared with the standard strains, the biofilm mass was significantly decreased in the $\triangle c l p P$ mutant strains at 12,24 , and $48 \mathrm{~h}$. The biofilm formation ability of the complement strains was restored with no significant difference compared to the standard strains $(p<0.01 ;$ Figure $3 b)$.

\section{ClpP depletion inhibited cell viability}

CLSM was used to determine the cell viability and biofilm mass from $6 \mathrm{~h}$ to $48 \mathrm{~h}$. The three groups were dominated by live bacterial cells, whereas the number of bacteria in the $\triangle c l p P$ mutant strains gradually increased with an increased proportion of dead bacteria compared to the other strains. Large biofilm sheets were observed in the standard and clpP complement strains cultures from 24 to $48 \mathrm{~h}$; however, smaller biofilm sheets were generated by the $\triangle c l p P$ mutant strains over the same time. These differences were statistically significant $(p<0.01$; Figure $3 c$ and $3 d$ ).

\section{ClpP deletion reduced the polysaccharide matrix mass}

CLSM detected the mass of the polysaccharide matrix to further explore the biofilm-forming capacity of the $E$. faecalis strains. Results show that the amount of polysaccharide matrix increased from $6 \mathrm{~h}$ to $48 \mathrm{~h}$ in all three strains. However, compared to the standard

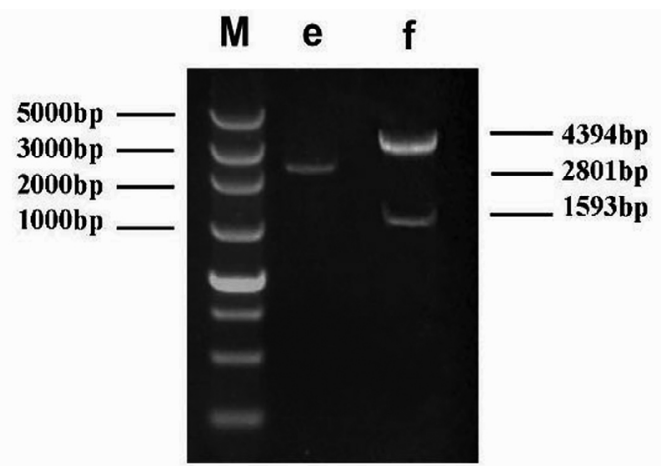

Figure 2- Identification of the clpP deletion strains and clpP complement strains. M: DNA marker; a) fragment of the clpP gene; b) PCR product of pUC18- $\Delta$ clpP-ermB-clpP; c) PCR product of pUC18- $\Delta$ clpP-ermB; d) PCR product of the pUC18- $\Delta$ clpP; e) fragment of $\Delta$ clpPermB; f) fragment of $\Delta$ clpP-ermB-clpP 
strains and complement strains, the mass of the polysaccharide matrix in the $\triangle c l p P$ mutant strains was lower at all time points (Figure 4 ).
Characterization of the biofilm microstructure

The biofilm microstructure conditions were observed by SEM (Figure 5). After 24 h, the standard and $c / p P$ complement strains formed a mature

a
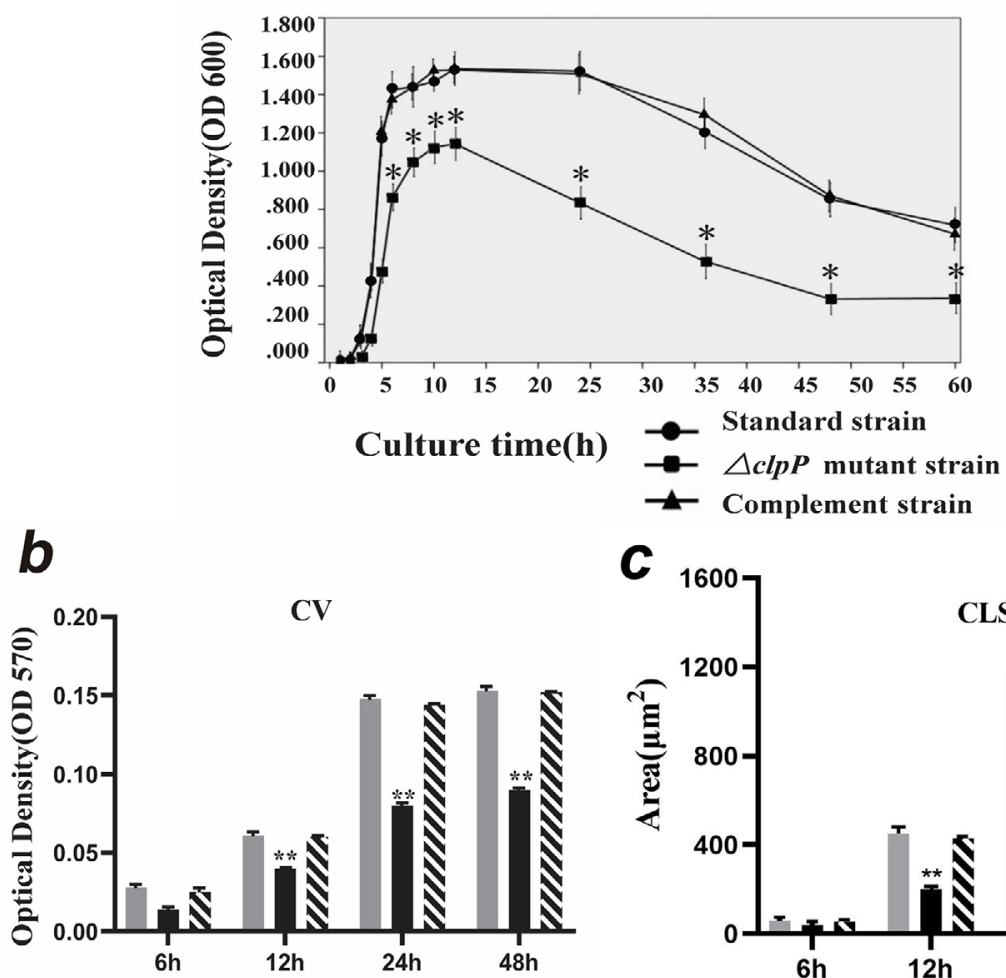

c
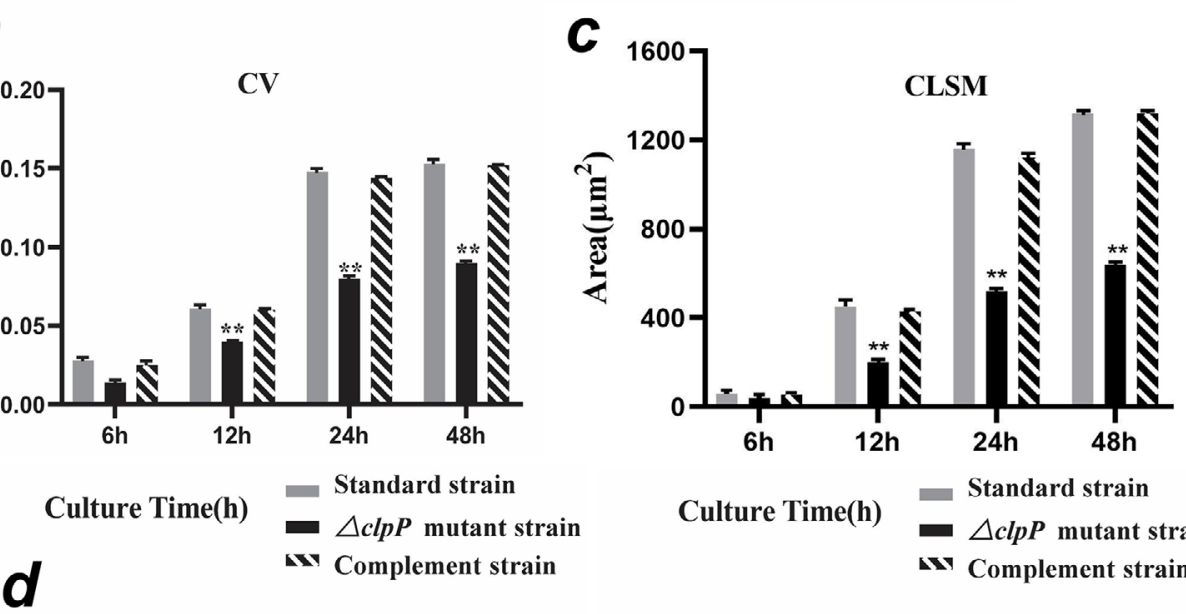
Culture Time(h)
- Standard strain
- $\triangle$ clpP mutant strain
wr Complement strain
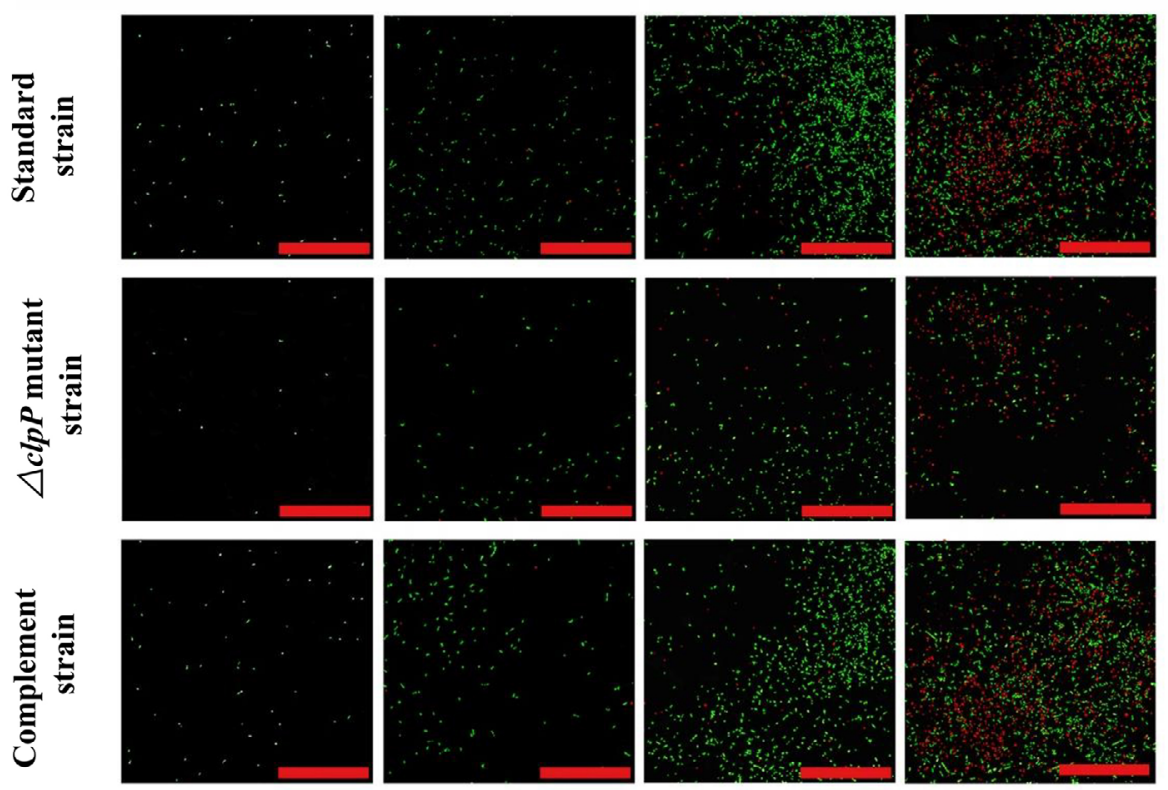

Figure 3- ClpP protease deletion inhibited growth and biofilm mass of $E$. faecalis. a) clpP gene deletion inhibits the growth of $E$. faecalis. The $\Delta$ clpP mutant strains exhibits impaired growth compared with the standard and clpP complement strains $(p<0.05)$. b) Crystal violet staining shows that the biofilm mass in the $\triangle$ clpP mutant strains decreased at 12,24 , and $48 \mathrm{~h}(\mathrm{p}<0.01)$ compared to the standard strains. c) CLSM examination of biofilm formation mass. The biofilm area in the $\Delta$ clpP mutant strains is smaller than that in the standard and complement strains at 12,24 , and $48 \mathrm{~h}(\mathrm{p}<0.01)$. d) CLSM to observe the cell viability. The number of $\Delta$ clpP mutant bacteria increased slowly, and the proportion of dead bacteria (red) was greater than the standard and complement strains (green fluorescent signal: staining by SYT90; red fluorescent signal: staining by PI; scale bar $=50 \mu \mathrm{m}$ ) 
biofilm structure (magnification $\times 1,000, \times 5,000$, and $\times 10,000)$ comprising grainy secretions and filaceous links with no significant difference observed between the strains. Meanwhile, the $\triangle c l p P$ mutant strains had a lower biofilm volume and a more regular shape.

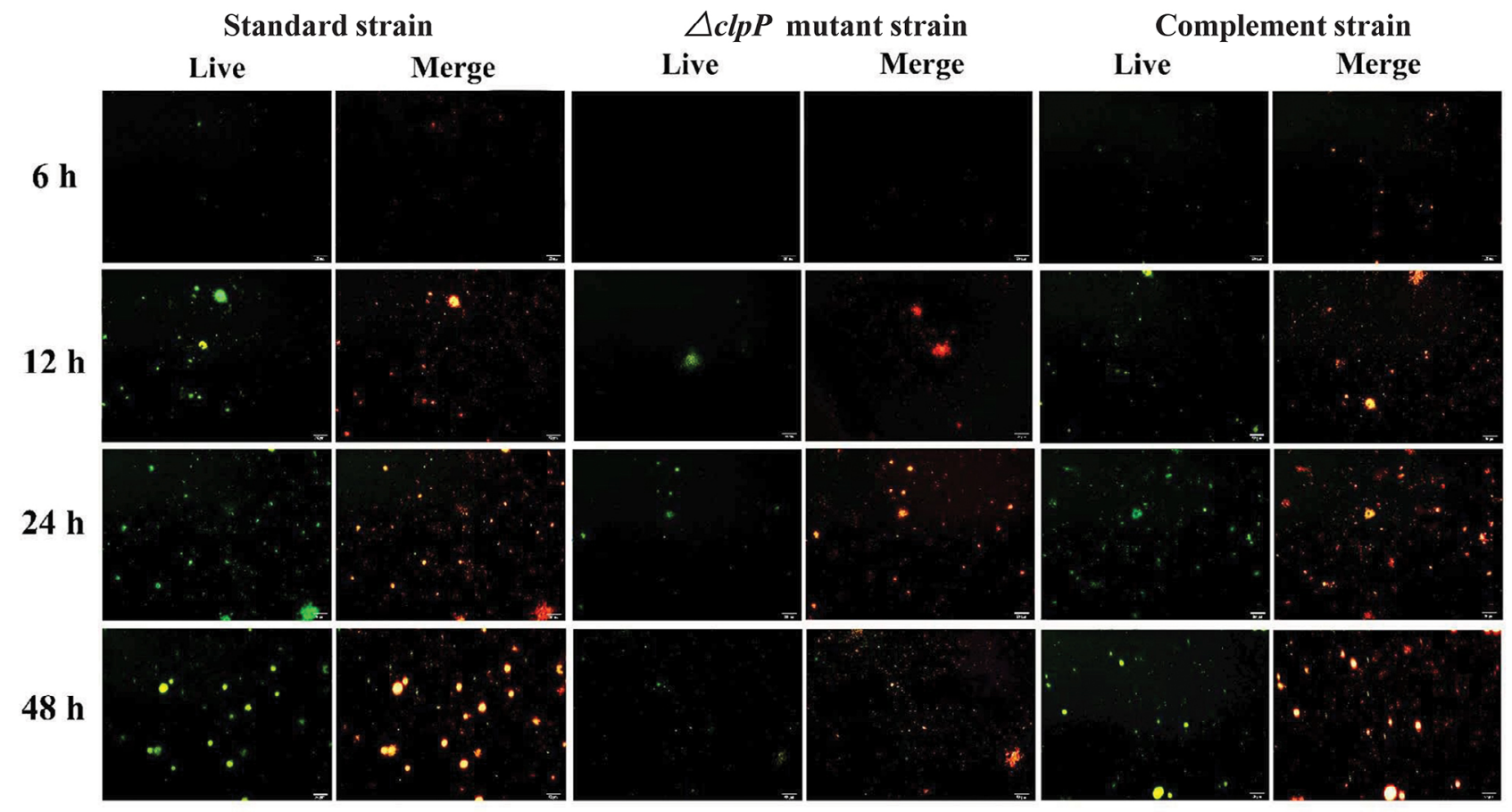

Figure 4- ClpP deletion reduced the polysaccharide matrix mass. Compared to the standard and complemented strains, the mass of polysaccharide matrix in the $\Delta \mathrm{clpP}$ mutant strains were decreased at $6,12,24$, and $48 \mathrm{~h}$ (Live: staining by FITC-ConA; Merge: staining by FITC-ConA and PI. scale bar $=20 \mu \mathrm{m}$ )
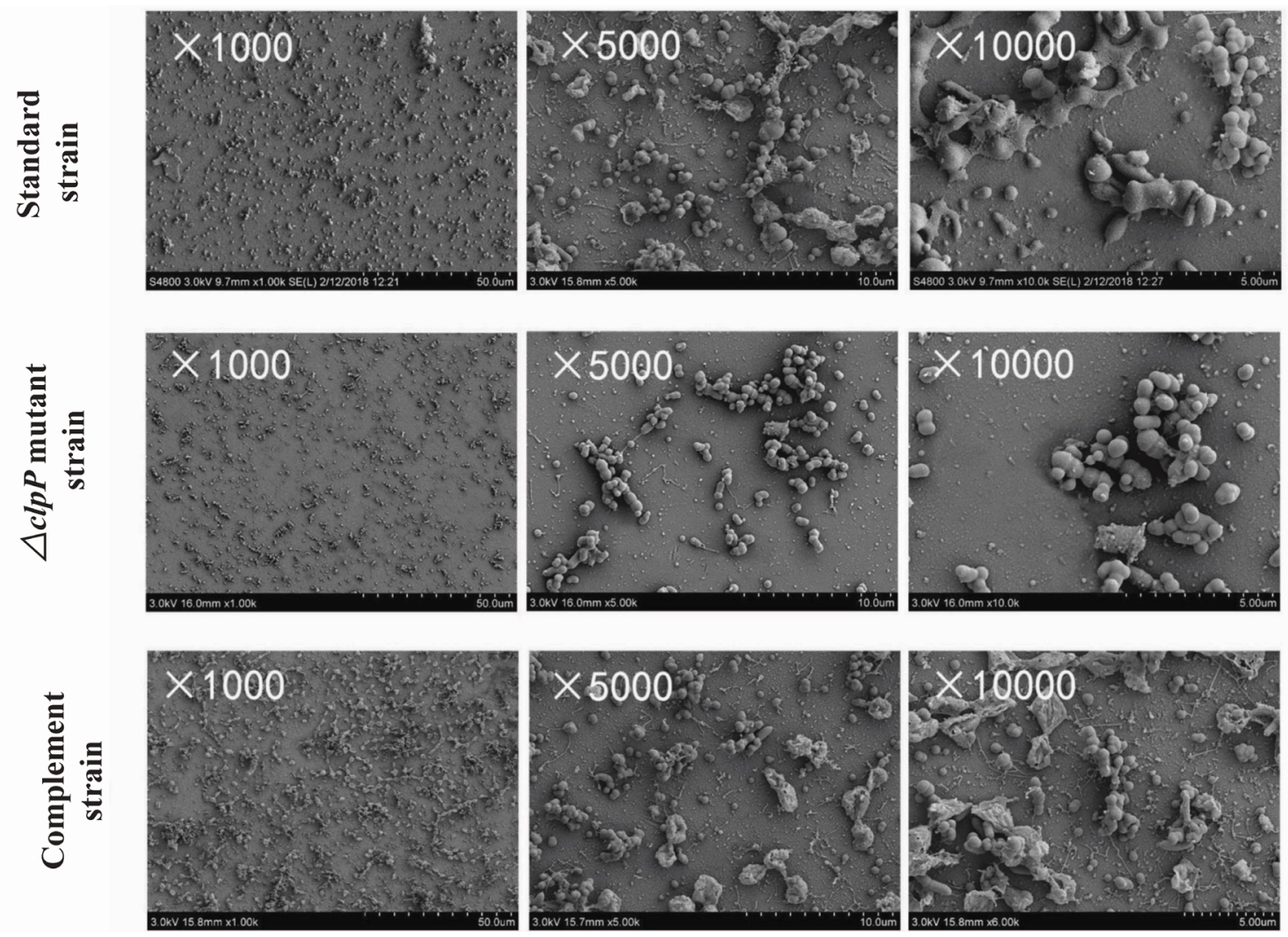

Figure 5- Biofilm microstructure conditions observed by scanning electron microscopy at $24 \mathrm{~h}$. SEM results show that the $\triangle$ clpP mutant strains had a lower biofilm volume and a more regular shape (magnification $\times 1,000, \times 5,000$, and $\times 10,000$ ) 


\section{Discussion}

ClpP is a key member of the Clp family and participates in the hydrolysis of misfolded and defective proteins. For many pathogenic bacteria, such as Listeria monocytogenes, Salmonella typhimurium, Streptococcus mutants, and Staphylococcus aureus, deletion of the $c / p P$ gene reduces pathogenicity, indicating that ClpP plays an important role in these bacteria. ${ }^{19-21}$ Studies on clpP can, therefore, help us to further explore the pathogenic mechanism of various bacteria. To date, few studies have examined if ClpP participates in the survival of $E$. faecalis or enhances its ability to form biofilm.

Studies on $E$. faecalis are important for understanding the pathogenesis of biofilms, which are surface-attached microbial communities embedded in extracellular polymeric substances. ${ }^{22-23}$ One factor affecting the formation of biofilms is the quantity of proliferated bacteria. In our study, strains with the $c l p P$ gene deleted, and $c / p P$ complement strains were constructed using homologous recombination technology, which requires only one round of recombination to achieve gene insertion and mutation. Gene knockout enabled us to test the specific function of the $c / p P$ gene, which can base subsequent studies on biofilm formation. The proliferative capacity of the three strains was then observed continuously using a classic viable colony counting method, which is simple yet reliable and has a low associated cost. The results showed that the standard strains had a similar variation tendency as the clpP complement strains, with a quiescent stage of approximately 10 $\mathrm{h}$ to $24 \mathrm{~h}$. However, the growth pattern of the clpP deletion strains was significantly altered with a shorter quiescent stage and impaired growth compared to the standard and complement strains. Moreover, the polysaccharide matrix, an important component of biofilms, was examined. Polymeric substances are not only necessary for bacterial adhesion, but also serve as a cytoskeleton in biofilm formation, which is beneficial for the survival of bacteria in this malnourished environment. ${ }^{24-26}$ The results show that the polysaccharide matrix mass in the $c / p P$ deleted strains was decreased compared with standard and complement strains.

The changes in growth pattern and the decrease in polysaccharide matrix may explain the $\mathrm{CV}$ and fluorescence staining results for the $c / p P$ deletion strains, which were examined using SEM and CLSM. These methods are effective for analyzing biofilms both quantitatively and qualitatively. The standard and complemented strains were observed to contain a large number of live bacteria during the quiescent stage $(24 \mathrm{~h})$. With an increased time, the biofilm area increased slowly, but the proportion of dead bacteria also significantly increased. In contrast, growth of the $c / p P$ deletion strains was inhibited. After the quiescent stage, the biofilm also gradually increased, but the general level was much lower than that of the standard and complement strains. It was also confirmed morphologically by SEM that mature biofilms were formed in the standard strains after $24 \mathrm{~h}$, whereas the biofilm formed by the $c l p P$ deletion strains was loose. CLSM further showed the formation of a large biofilm sheet by the standard and $c / p P$ complement strains; however, only a small and dispersive biofilm was formed by the $c l p P$ deletion strains.

The results in our study are consistent with those observed in other Gram-positive bacteria. For example, Wang, et al. ${ }^{27}$ (2007) found that $c l p P$ influences the initial attachment of bacteria to decrease biofilm formation of Staphylococcus Epidermidis. This result is also consistent with our previous findings in the Gramnegative $P$. gingivalis. ${ }^{15}$ Studies with Streptococcus mutans have also reported that $c / p P$ gene deletion increases biofilm formation in Gram-positive bacteria under special environments. ${ }^{28}$ Based on these results, we hypothesized that the $c / p P$ gene may have different regulatory effects in different bacteria. However, a bacteria being either Gram-positive or Gram-negative does not appear to be a criterion for defining ClpP effects.

The role of ClpP in E. faecalis has been rarely studied. Zheng, et al. ${ }^{29}$ (2020) found that $c / p P$ inhibits the formation of $E$. faecalis biofilms, although it has no effect on the growth of $E$. faecalis. This differs from our results, which showed that $c / p P$ expression was also closely related to growth. Studies have also found that $c / p P$ influenced growth and filament formation Salmonella Enterica Serovar Typhimurium at low temperature. ${ }^{30}$ Moreover, autolysis has been shown to play an important role in bacterial development, including biofilm formation. ${ }^{31}$ Researchers found that $\mathrm{ClpC}$, another member of the Hsp100/ ATPase family, could release autolysin A to alter its growth ability. ${ }^{32-33}$ Further investigation is, therefore, required to determine if $c / p P$ can alter the proliferative capacity 
and biofilm formation in E. faecalis by autolysis.

Considering that $E$. faecalis is one of the main etiologic pathogens for root canal reinfection and persistent periapical periodontitis, whereas also exhibiting a certain resistance to most root canal therapy drugs, cleaning and disinfection methods, ${ }^{34}$ the results of the current study may provide insights into novel targets to better control $E$. faecalis infections. Thus, we showed that ClpP can increase the biofilm formation of $E$. faecalis. We also demonstrated that ClpP may serve as a potential therapeutic target for $E$. faecalis. Recently, acyldepsipeptides have been identified as a new antibiotic that targets ClpP to elicit a bactericidal role. Hence, application of acyldepsipeptides as a targeted drug for ClpP in root canal therapy could also be effective. Nevertheless, elucidation of the mechanism by which ClpP affects biofilm formation and growth of $E$. faecalis is necessary in future studies. ${ }^{35}$

\section{Conclusions}

Our studied shows that ClpP can increase biofilm formation in E. faecalis and emphasizes the importance of ClpP as a potential target against $E$. faecalis.

\section{Acknowledgments}

This study was supported by grants from the National Nature Science Foundation of China (grant number 81500833 ).

\section{Author disclosure statement}

The authors declare no conflicts of interest.

\section{Authors' contributions}

Feng, Ying: Conceptualization (Equal); Data curation (Equal); Investigation (Equal); Visualization (Equal); Writing-original draft (Equal). Wang, Hongyuan: Formal analysis (Equal); Resources (Equal). He, Lu: Investigation (Equal); Software (Equal). Liu, Yi: Project administration (Equal); Supervision (Equal). Li, Hong: Conceptualization (Equal); Funding acquisition (Equal); Methodology (Equal); Writing-review \& editing (Equal).

\section{References}

1- Bouillaguet S, Manoil D, Girard M, Louis J, Gaïa N, Leo S, et al. Root microbiota in primary and secondary apical periodontitis. Front Microbiol. 2018;9:2374-84. doi: 10.3389/fmicb.2018.02374

2- Zhang C, Du J, Peng Z. Correlation between Enterococcus faecalis and persistent intraradicular infection compared with primary intraradicular infection: a systematic review. J Endod. 2015;41(8):1207-13. doi: 10.1016/j.joen.2015.04.008

3- Zheng JX, Wu Y, Lin ZW, Pu ZY, Yao WM, Chen Z. Characteristics of and virulence factors associated with biofilm formation in clinical Enterococcus faecalis isolates in China. Front Microbiol. 2017; 8:2338. doi: $10.3389 /$ fmicb. 2017.02338

4- Bowen WH, Burne RA, Wu H, Koo H. Oral biofilms: pathogens, matrix, and polymicrobial interactions in microenvironments. Trends Microbiol. 2018;26(3):229-42. doi: 10.1016/j.tim.2017.09.008

5- Khalifa L, Shlezinger M, Beyth S, Haddad YH, Glazer SC, Beyth N, et al. Phage therapy against Enterococcus faecalis in dental root canals. J Oral Microbiol. 2016;8:32157-67. doi: 10.3402/jom. v8.32157 6- Lei L, Shao M, Yang Y, Mao MY, Yang YM, Hu T. Exopolysaccharide dispelled by calcium hydroxide with volatile vehicles related to bactericidal effect for root canal medication. J Appl Oral Sci. 2016;24(5):487-95. doi: 10.1590/1678-775720160014

7- Riboulet E, Verneuil N, La Carbona S, Sauvageot N, Auffray Y, Hartke $A$, Relationships between oxidative stress response and virulence in Enterococcus faecalis. J Mol Microbiol Biotechnol. 2007;13(1-3):140-6. doi: $10.1159 / 000103605$

8- Oliveira NE, Abranches J, Gaca AO, Laport MS, Damaso CR, Bastos $\mathrm{MC}$, et al. ClpB, a class III heat-shock gene regulated by CtsR, is involved in thermotolerance and virulence of Enterococcus faecalis. Microbiology (Reading). 2011;157(Pt 3):656-65. doi: 10.1099/ mic.0.041897-0

9- Szczepanowska K, Maiti P, Kukat A. Hofsetz E, Nolte H, Senft K, et al. CLPP coordinates mitoribosomal assembly through the regulation of ERAL1 levels. EMBO J. 2016;35(23):2566-83. doi: 10.15252/ embj. 201694253

10- Foster TJ. Antibiotic resistance in Staphylococcus aureus: current status and future prospects. FEMS Microbiol Rev. 2017;41(3):430-49. doi: $10.1093 /$ femsre/fux007

11- Brötz-Oesterhelt $H$, Sass P. Bacterial caseinolytic proteases as novel targets for antibacterial treatment. Int J Med Microbiol. 2014;304(1):23-30. doi: 10.1016/j.ijmm.2013.09.001

12- Lemos JA, Burne RA. Regulation and physiological significance of ClpC and ClpP in Streptococcus mutans. J Bacteriol. 2002;184(22):635766. doi: $10.1128 / \mathrm{jb} .184 .22 .6357-6366.2002$

13- Hill NS, Zuke JD, Buske PJ, Chien AC, Levin PA. A nutrientdependent division antagonist is regulated post-translationally by the Clp proteases in Bacillus subtilis. BMC Microbiol. 2018;18:29-42. doi: 10.1186/s12866-018-1155-2

14- Xie F, Zhang Y, Li G, Zhou L, Liu SG, Wang CL. The ClpP protease is required for the stress tolerance and biofilm formation in Actinobacillus pleuropneumoniae. PLoS One. 2013;8(1):e5360. doi: 10.1371/journal. pone. 0053600

15- He L, Wang H, Zhang R, Li H. The regulation of Porphyromonas gingivalis biofilm formation by ClpP. Biochem Biophys Res Commun. 2019;509(2):335-40. doi: 10.1016/j.bbrc.2018.12.071

16- Liu Q, Wang X, Qin J, Cheng S, Yeo WS, He L, et al. The ATPdependent protease ClpP inhibits biofilm formation by regulating agr and cell wall hydrolase Sle1 in Staphylococcus aureus. Front Cell Infect Microbiol. 2017;7:181. doi: 10.3389/fcimb.2017.00181

17- Shanks RM, Caiazza NC, Hinsa SM, Toutain CM, O'Toole GA. Saccharomyces cerevisiae-based molecular tool kit for manipulation of genes from gram-negative bacteria. Appl Environ Microbiol. 2006;72(7):5027-36. doi: 10.1128/AEM.00682-06 
18- Hall BM, Breidenstein EB, Reffuveille F, Mawla GD, Hancock $\mathrm{RE}$, Baker $\mathrm{TA}$, et al. Two isoforms of Clp peptidase in Pseudomonas aeruginosa control distinct aspects of cellular physiology. J Bacteriol. 2017;199(3):e00568-16. doi: 10.1128/JB.00568-16.

19- Frees D, Chastanet A, Qazi S, Sørensen K, Hill P, Msadek T, et al. Clp ATPases are required for stress tolerance, intracellular replication, and biofilm formation in Staphylococcus aureus. Mol Microbiol. 2004;54(5):1445-62. doi: 10.1111/j.1365-2958.2004.04368.x

20- Kwon HY, Kim SW, Choi MH, Ogunniyi D, Paton JC, Park SH, et al. Effect of heat shock and mutations in $\mathrm{ClpL}$ and $\mathrm{ClpP}$ on virulence gene expression in Streptococcus pneumoniae. Infect Immun. 2003;71(7):3757-65. doi: 10.1128/iai.71.7.3757-3765.2003

21- Michel A, Agerer F, Hauck CR, Herrmann M, Ullrich J, Hacker J, et al. Global regulatory impact of ClpP protease of Staphylococcus aureus on regulons involved in virulence, oxidative stress response, autolysis, and DNA repair. J Bacteriol. 2006;188(16):5783-96. doi: 10.1128/JB.00074-06

22- Tendolkar PM, Baghdayan AS, Shankar N. Putative surface proteins encoded within a novel transferable locus confera high-biofilm phenotype to Enterococcus faecalis. J Bacteriol. 2006;188(6):2063-72. doi: $10.1128 /$ JB.188.6.2063-2072.2006

23- Yamaguchi M, Noiri Y, Kuboniwa M, Yamamoto R, Asahi Y, Maezono $\mathrm{H}$, et al. Porphyromonas gingivalis biofilms persist after chlorhexidine treatment. Eur J Oral Sci. 2013;121(3 Pt 1):162-8. doi: 10.1111/ eos. 12050

24- Ramos Y, Rocha J, Hae AL, van Gestel J, Vlamakis H, Bentley CC, et al. PolyGlcNAc-containing exopolymers enable surface penetration by non-motile Enterococcus faecalis. PLoS Pathog. 2019;15(2):e1007571. doi: 10.1371/journal.ppat.1007571

25- Yu MK, Kim MK, Rosa V, Hwang YC, Fabbro MD, Sohn WJ, et al. Role of Extracellular DNA in Enterococcus faecalis biofilm formation and its susceptibility to sodium hypochlorite. J Appl Oral Sci. 2019;27:e20180699. doi: 10.1590/1678-7757-2018-0699

26- Li W, Liu HY, Xu Q. Extracellular dextran and DNA affect the formation of Enterococcus faecalis biofilms and their susceptibility to $2 \%$ chlorhexidine. J Endod. $2012 ; 38(7): 894-8$. doi: $10.1016 / \mathrm{j}$. joen.2012.04.007
27- Wang CZ, Li M, Dong DD, Wang JP, Ren J, Otto M, et al. Role of ClpP in biofilm formation and virulence of Staphylococcus epidermidis. Microbes Infect. 2007;9(11):1376-83. doi: 10.1016/j. micinf.2007.06.012

28- Zhang JQ, Hou XH, Song XY, Ma XB, Zhao YX, Zhang SY. ClpP affects biofilm formation of Streptococcus mutans differently in the presence of cariogenic carbohydrates through regulating gtfBC and ftf. Curr Microbiol. 2015;70(5):716-23. doi: 10.1007/s00284-015-0779-9 29- Zheng JX, Wu Y, Lin ZW, Wang GF, Jiang S, Sun X, et al. ClpP participates in stress tolerance, biofilm formation, antimicrobial tolerance, and virulence of Enterococcus faecalis. BMC Microbiol. 2020;20(1):30. doi: 10.1186/s12866-020-1719-9

30- Knudsen GM, Nielsen MB, Thomsen LE, Aabo S, Rychlik L, Olsen JE. The Role of ClpP, RpoS and CsrA in growth and filament formation of Salmonella enterica serovar Typhimurium at low Temperature. BMC Microbiol. 2014;14:208. doi: 10.1186/s12866-014-0208-4

31- Thomas VC, Hancock LE. Suicide and fratricide in bacterial biofilms. Int J Artif Organs Actions. 2009;32(9):537-44. doi: 10.1177/039139880903200902

32- Lbrahim YM, Kerr AR, Silva NA, Mitchell TJ. Contribution of the ATP-dependent protease ClpCp to the autolysis and virulence of Streptococcus pneumoniae. Infect Immun. 2005;73(2):730-40. doi: 10.1128/IAI.73.2.730-740.2005

33- Chatterjee L, Maisonneuve E, Ezraty B, Herrmann M, Dukan S. Staphylococcus aureus $\mathrm{ClpC}$ is involved in protection of carbonmetabolizing enzymes from carbonylation during stationary growth phase. Int J Med Microbiol. 2011;301(4):341-6. doi: 10.1016/j. ijmm.2010.10.002

34- Stuart $\mathrm{CH}$, Schwartz SA, Beeson TJ, Owatz CB. Enterococcus faecalis: its role in root canal treatment failure and current concepts in retreatment. J Endod. 2006;32(2):93-8. doi: 10.1016/j. joen.2005.10.049

35- Mabanglo MF, Leung E, Vahidi S, Seraphim TV, Eger BT, Bryson S, et al. ClpP protease activation results from the reorganization of the electrostatic interaction networks at the entrance pores. Commun Biol. 2019;2:410. doi: 10.1038/s42003-019-0656-3 